\title{
An essay on the use of new antipsychotics
}

\author{
Robert W. Kenwin
}

In a textbook of psychopharmacology published as recently as 1990, Hollister and Csernansky wrote about antipsychotics "It is most discouraging that more effective pharmacotherapy has not been developed. Present drugs have many defciences: they are not curative; their ameliorative effects are often limited, many patients remain totally unresponstve; they are unpleasant to take so that many patients are less than fully compliant; they produce major side effects such as tardive dyskinesia whose full implications are still uncertain".

Shortly after this was published clozapine was introduced into the UK and USA for treatment resistant schizophrenia-a drug with a good efficacy and low side effect profile (Kane et al 1988) and in summer 1993 risperidone was introduced into the UK with probably superior efficacy than haloperidol, with fewer Parkinsonian side effects (Marder, 1994).

No reasonable psychiatrist would have cause to doubt Hollister and Csernansky's assertions, yet in the UK, the enthusiasm for the use of new atypical antipsychotics has not been as great as one might expect, with only 3500 patients on clozapine, and risperidone often inappropriately being reserved as second line therapy. The reasons for this are probably several fold: cost; the false perception of new drugs as dangerous: for clozapine, too strict a definition of treatment resistance; for risperidone, reserving its use in a clinical decision free after first line treatments have been tried; finally, many psychiatrists are concerned about compliance and prefer depot medication. If cost and doubts about safety were not an issue and leaving aside the need for sedation, the ideal treatment protocol for a first onset case of schizophrenia might be to try risperidone first and if patients prove resistant or intolerant after a trial of risperidone to transfer them on to clozapine. This is clearly impracticable and this article attempts to debate some of these issues.

\section{Cost}

The actual average cost for a one year course of clozapine is about $£ 1800$. The estimated cost to the hospital, taking into account monitoring arrangements and centralisation of factities, has been estimated at $\$ 2500$ per annum (Matheson et al, 1993). The annual costs of risperidone are estimated at $£ 900$ p.a. Since clozapine is reserved for treatment resistant patients, a comparable cost might be that of a long acting depot such as Depixol which, on average, costs $£ 353$ p.a. Since risperidone is for acute illnesses and relapses a comparable drug might simply be chlorpromazine or haloperidol at a cost of $£ 288$ p.a. Are the costs of these drugs, therefore, ever justified on clinical and cost grounds? About 20$30 \%$ of patients are treatment resistant (Kane et al, 1988) and are potential candidates for clozapine. In Kane et ails (1988) trial, about 30\% of the patients responded. However, these patients had very chronic illness. In the UK. response rates of about $70 \%$ are seen (King \& Mills 1993; Shaikh et al, 1994). The magnitude of response and lack of side effects is a justifiable argument alone - but is the drug cost effective? Meltzer \& Cola (1994), reviewing three published pharmacoeconomic studies of clozapine, found that improvements in psychopathology could easily be correlated with cost utility savings. Revickd et al (1990) studied 133 clozapine treated patients compared with 51 typical neuroleptic treated patients in the US. The clozapine group had a lower Brief Psychiatric Rating Scale (BPRS) score and lower rehospitalisations (26\%) compared to the typical group (56\%) over a two year period. Direct costs for the clozapine group were $\$ 80440$ in the 12 months prior to treatment and $\$ 55867$ in the subsequent 12 months following treatment. This was $\$ 16000$ less than the typical neuroleptic treated cohort. This study has been criticised for excluding patients who remained undischarged and clozapine drop-outs from the analysis. Meltzer et al (1993) performed a two year pre- and post-clozapine cost evaluation in a cohort of 96 patients. The clozapine patients were significantly improved on the BPRS, Clinical Gobal Impressions scale (CGI) and quality of life and were more able to participate in work and domestic life than before. Total costs for the clozapine group decreased from \$71798 per two years pre-clozapine to $\$ 25906$ in the two years on clozapine - a saving of \$45892 entirely 
accounted for by hospital costs. In a further study by Reid et al (1994) of a severely ill Texan cohort, savings per patient per year were $\$ 33000$ to $\$ 50250$. In a commentary, Reid (1994) writes "If there is no new money - and there usually is not - we must insist that mental health directors and agency boards restructure priorities for existing health funds to reflect the importance of new and more effective drugs - it's the right thing to do".

Risperidone is about half the cost of clozapine. Its direct value for money in terms of clinical benefit is uncomparable in the UK because of the different indications and licensing structure. As yet, no formal cost-utility assessment of risperidone has been undertaken and there is clearly a need for this. If the clinical trial successes are reflected in clinical practice, as with clozapine, the financial implications of treatment with risperidone are likely to be offset with long-term advantages such as reduced hospital admissions, improved compliance and a reduction in debilitating side effects.

\section{Safety}

There is a general belief that new antipsychotics are unsafe. This is an artefact of rigld postmarketing survelllance to which new drugs are subjected and from which older drugs are immune. The neutropenia and agranulocytosis with clozapine are well known, but the Clozaril patient monitoring service neutralises this. The predicted number of cases does not arise because of monitoring and the rate of detected neutropenia and agranulocytosis is $6 \%$ and $0.4 \%$ respecttvely. There have been two deaths from clozapine-related agranulocytosis. This represents far fewer deaths on clozapine than other neuroleptic deaths. Jasik \& Lader (1994) have summarised the problem of unexpected neuroleptic deaths.

While little is known about the frequency of neutropenia and other adverse reactions with risperidone, older drugs are less effective and more liable to cause neurological, endocrine and autonomic side effects. Phenothazines are additionally associated with arrythmias, hepatic abnormalities, agranulocytosis, thrombocytopenia. convulsions, neuroleptic malignant syndrome, myocarditis, severe tardive dyskinesia and sudden death. The frequency of agranulocytosis with chlorpromazine is $1 / 1300$ and the neutropenia rate is $0.7 \%$ (Vincent, 1986). This is likely to be an underestimate as many reactions are cryptic and under-reported. It is the case, therefore, that the burden of risk is probably greater with older antipsychotics. Despite the intense focus new drugs come under, a balanced view of the adverse event profile of drugs shows older drugs are less safe compared to carefully monitored patients on newer drugs.

\section{Protocols, treatment resistance and clozapine}

There is mounting pressure for a careful evaluation of treatment resistance before clozapine is used, principally where the case for treatment is partly an economic one under budgetary pressure. To pick over details of what level of neuroleptic exposure defines resistance is a moot point. There is no subtype of schizophrenta represented as treatment resistant type for which clozapine is peculiarly useful. Clozapine is effective across the board in schizophrenia (e.g. Claghorn et al, 1987) and the point of its use which should guide the timing - is whether there is a subset of particularly disabled patients in whom the drug may justify the risk of agranulocytosis and the inconvenience of monitoring.

There is no point then in predicting clozapine responders by pursuing complex and megadose treatment protocols as this is already probably a deserving group in whom this tactic inflicts many adverse effects with very little statistical chance of clinical response. The scientific literature would support this axiomatic view. For instance, Baldessarini et al (1988) have concluded that neuroleptic regimes of greater than $600 \mathrm{mg}$ of chlorpromazine per day are associated with worse overall outcome. Several studies have reported that optimal doses may be in the lower rather than higher dose range (Rifkind et al, 1991). In a review of high and very high dose antipsychotics, Aubree \& Lader (1980) concluded that there was little to be gained from high dose antipsychotics and a great deal to be lost in terms of neurological, endocrine and cardiovascular side effects. Most recently, the Royal College of Psychiatrists (Thomson, 1994) came to the same conclusions and Jasik \& Lader (1994) claim to have identified a number of fatalities associated with high dose phenothiazines. Functional imaging studies also support this view. In our own studies (Pllowsky et al, 1993), we have shown that resistant patients remain so despite full $\mathrm{D} 2$ receptor blockade and that D2 receptors are fully blocked across a wide range of low to modest doses. Furthermore, all classes of classical antipsychotics fully block D2 receptors at modest doses (Pllowsky et al, 1993). Therefore, there is nothing to be gained pharmacodynamically by pressing on with high dose or varying drug regimes. Whatever treatment protocols are adopted locally, it is probably best to base them on a philosophy of clinical optimisation around low to modest doses of classical drugs, rather than pursuing a multidrug or high dose regime, and to use clozapine earlier, rather than 
inflicting intense discomfort on patients in the academic pursuit of treatment resistance.

\section{Risperidone: first line or second line}

Risperidone is licensed without restriction and may well be a valuable drug in first onset schizophrenia, where it is effective, better tolerated than older drugs and has a lower side effect profile (Marder, 1994). However, as with many new drugs, it is often reserved as second or third line treatment after a course of tried and tested treatment. This is illogical, as the latency associated with attempts at treatment may, of itself, predispose to poorer response to drugs (Johnstone et al, 1993). Also, such a procedure selects treatment resistant patients only for subsequent risperidone and militates against response. Anyway, wouldn't such patients be fulfilling criteria for clozapine treatment and be better off for it? It would be useful to know how risperidone fared as a second line treatment by comparing it with clozapine in treatment resistance. However, no such study has been performed and clozapine remains the gold standard for treatment resistance.

\section{Compliance and new drugs}

Compliance is a major factor in determining the continuance of new antipsychotics. As Hale (1993) pointed out, depot neuroleptics remain the best authenticated method in non compliant. forgetful or treatment resistant patients (Tegeler et al, 1980). There is, however, very little objective data on compliance with oral antipsychotics and there is a strong lobby for depot atypicals. This, of course. would be precluded for clozapine but is theoretically possible, although chemically diffcult, for risperidone. The major barriers to compliance are extrapyramidal side effects (Frances \& Weiden, 1987) and lack of insight (Buchanan, 1992). One would imagine that these two difficulties may be obviated in drugs such as clozapine and risperidone, with superior efficacy and a low extrapyramidal side effects, profile. Statements such as "the delivery system most likely to offer the long term benefits of new drugs to patients with recalcitrant chronic schizophrenia is the well tried depot system" (Hale, 1993) need challenging with objective clinical trial data. particularly since clozapine will never be available in this format. In our own studies of previously non compliant patients (Aitchison \& Kerwin. 1994) we have followed up a series of 24 patients over a two year period on oral clozapine with no compliance difficulties and a relapse rate of $2 / 24$. Extrapyramidal symptoms were markedly diminished and insight and quality of life significantly improved. Whether these or other factors encourage compliance require formal testing.

\section{References}

Artchison, K. J. \& KERWIN, R. W. (1994) The costs and benefits of clozapine therapy in the UK (Abstract). Schizophrenia Research, 16, 141.

AUBREE, J. C. \& LADER, M. H. (1980) High and very high dose antipsychotics: a critical review. Journal of Clinical Psychiatry, 41, 341-356.

Baldessarin, R. J., Cohen, B. M. \& Teicher, M. H. (1988) Significance of neuroleptic doses and plasma levels in the pharmacological management of the psychoses. Archives of General Psychiatry, 46, 79-91.

Buchanan, A. (1992) A two year prospective study of treatment compliance in patients with schizophrenia. Psychological Medictne, 29, 787-789.

CLAGHORN, J., Hongreid. G. ABUzanha, S. S., et al (1987) The risks and benefits of cloceapine versus chlorpromartine. Joumal of Cinical Psychopharmacology, 7, 377-384.

Frances, A. J. \& WEIDEN, P. (1987) Promoting compliance with outpatient drug treatment. Hospital and Community Psychiatry. Se, 1158-1160.

HALE, A. (1993) Will the new antipsychotics improve the treatment of schizophrenia. Brittsh Medical Joumal. 307, 749-750.

Hounster, L. E. \& CSERnAnskY, J. G. (1990) Clinical Pharmacology of Psychotherapeutic Drugs (3rdedn). New York: Churchill Ltvingstone.

JASIK. N. \& LADER, M. (1994) Post-mortem antipsychotic drug concentrations and unexplained deaths. Brttish Joumal of Psychiatry, 166, 787-791.

JOHNSTONE, E. C., et al (1993) Schizophrenia: problems in clinical practice. Lancet, $\mathbf{3 4 1}$, 536-538.

KANe, J., Hongfeid, G., SINGER, J., et al (Clozarll Collaborattve Study Group) (1988) Cloceapine for the treatment resistant schizophrenic: a double blind comparison with chlorpromazine. Archives of General Psychiatry, 46, 789-796.

King, D. J. \& Mins, P. (1993) Clozapine: the Holywell experience with the first 24 patients. Irtsh Joumal of Psychological Medictine, 10. 30-34.

MARDER, S. R. (1994) Risperidone in the treatment of schizophrenia. American Joumal of Psychiatry. 161. 825-835.

Matheson, L. A., CoOK. H. M., McKenna, P. J., et al (1993) Purchasing Care for Patlents with Schizophrenia. Health Policy Unit review No 5. England: Daniels Publishing

Meltzer, H. Y. \& COLA. P. A. (1994) The pharmacoeconomics of clozapine: a review. Joumal of Clinical Psychiatry. 85 (Suppl. B), 161-165.

-. COLA, P.. WAY, L., et al (1993) Cost-effectiveness of clozapine in neuroleptic resistant schizophrenia. American Journal of Psychiatry. 160, 1630-1638.

PILOWSKY, L. S., CostA. D. C., MURRAY, R. M., et al (1993) Antipsychotic medication, D2 dopamine receptor blockade and clinical response: a ${ }^{123} \mathrm{I}-\mathrm{IBZM}$ SPET (single photon emission tomography) study. Psychological Medicine, 23, 791-797.

REID, W. H., MASON, M. \& TOPRAL. M. (1994) Savings in hospital bed days related to treatment with clozapine. Hospital and Community Psychiatry. 46, 261-264.

- (1994) The treatment of psychosis: resetting the drug cost thermostat. Journal of Clinical Psychiatry. 56 (Suppl. B). 166-168.

REVICKI. D. A., LUCE, B. R., WeXHLER. J. M., et al (1990) Costeffecttveness of clozapine for treatment resistant schizophrenic patients. Hospital and Community Psychiatry. 41. 850-854. 
RIFKIND, A., DODDI, S., KARAGIAGI, B., et al (1991) Dosage of haloperidol for schizophrenia. Archives of General Psychiatry, 48, 166-170.

SHAKK, S., Couler. D., KERWIN, R. W., et al (1993) Dopamine D4 receptor subtypes and response to clozapine. Lancet. s41, 116.

THOMSON. C. (1994) The use of high-dose antipsychotic medication (Consensus Statement). British Journal of Psychiatry, 164, 448-458.
Vincent, P. C. (1986) Drug induced aplastic anaemia and agranulocytosis. Incidence and mechanisms. Drugs, 31. 52-63.

Robert W. Kerwin, Professor in Clinical Neuropharmacology and Psychiatry. Institute of Psychiatry, De Crespigny Park, London SE5 8AF

\title{
Commentary
}

\section{Response to Professor Kerwin}

\author{
T. R. E. Barnes
}

In the treatment of psychotic illness, clinicians regularly face the decision whether to prescribe a standard neuroleptic or a new, atypical drug. The relevant criterla for such a choice will vary depending on the clinical situation, and many of the data required for an informed assessment of the relattve risk-benefit balance for old and new drugs are not yet available. In his essay, Professor Kerwin's guiding theme is that atypical neuroleptics are not prescribed widely enough by clinicians, a view shared by other authorities in this area (Carpenter et al, 1995; Meltzer, 1995). The reasons he puts forward for this underuse include the high cost of these drugs, the false perception that new drugs are dangerous, too strict a definition of treatment resistance (in the case of clozapine), and the tendency for clinicians to reserve risperidone as a second-line treatment after standard neuroleptics have failed to produce a satisfactory response. In addition, some psychiatrists prefer depot medication for maintenance treatment, the principal advantage being the avoidance of covert non-compliance (Barnes \& Curson, 1995). Undoubtedly, these are all relevant factors in the degree of use of newer atypical neuroleptics. However, although it must be accepted that prescribing habits die hard, the degree to which the current reservations and caution of clinicians may be appropriate is a matter for discussion. Having been invited to comment on Kerwin's article, I would like to take the opportunity to elaborate on some of the issues raised.

\section{Side efiects and safety}

Concerning safety, Kerwin concludes that "a balanced view of the adverse event profile of drugs shows that older drugs are less safe compared to carefully monitored patients on newer drugs". While this may prove to be the case, it is uncertain whether the published evidence so far would support such an assertion. With regard to clozapine, any data relating to its use in the long term would refer only to those patients who had tolerated and responded to the medication: because of the increased risk of agranulocytosis there is a strong incentive to stop the drug in any patient who has shown a less than impressive response or developed problematic side effects. This might introduce a bias when comparing safety data for a cohort of patients receiving clozapine long term with similar groups on other neuroleptics. Furthermore, although a number of serious side effects are associated with standard neuroleptics (Barnes \& Edwards, 1993; Edwards \& Barnes, 1993), It is the evidence of an increased risk of a specific problem associated with a particular drug that tends to cause concern and leads to greater safety monitoring or restrictions on the patient population to whom it can be prescribed. The standard neuroleptics available would generally be thought to have similar profiles for both mild and severe side effects. Any differences observed may be partly related to clinicians' preferences for specific drugs or classes of drugs in particular 